\title{
EPIDEMIA DE COVID-19 NO BRASIL: UMA ANÁLISE DOS PRIMEIROS 50 DIAS
}

\author{
EPIDEMIC OF COVID-19 IN BRAZIL: \\ AN ANALYSIS OF THE FIRST 50 DAYS
}

Arthur de Almeida Medeiros (Orcid: 0000-0002-2192-8823) ${ }^{1,2}$

Isabele Ribeiro Barbosa (Orcid: 0000-0002-1385-2849)

Kenio Costa de Lima (Orcid: 0000-0002-5668-4398)2

Contato

Arthur de Almeida Medeiros

E-mail: arthur.medeiros@ufms.br

${ }^{1}$ Instituto Integrado de Saúde, Universidade Federal de Mato Grosso do Sul, Mato Grosso do Sul, Brasil.

${ }^{2}$ Programa de Pós-Graduação em Saúde Coletiva, Universidade Federal do Rio Grande do Norte, Rio Grande do Norte, Brasil.

Este trabalho teve apoio da Coordenação de Aperfeiçoamento de Pessoal de Nível Superior (CAPES), por meio do pagamento de bolsa de pós-doutorado a Arthur de Almeida Medeiros [processo 88887.372306/2019-00], e da Universidade Federal de Mato Grosso do Sul.

\section{RESUMO}

Objetivo: analisar a tendência temporal dos casos e óbitos por COVID-19 ocorridos no Brasil nos primeiros 50 dias da epidemia no país. Métodos: trata-se de estudo ecológico de série temporal com base nos registros de novos casos e óbitos ocorridos no período de 26 de fevereiro a 15 de março. Para análise da tendência de novos casos realizou Regressão Joinpoint, a partir da taxa acumulada diária, e também foram realizados os cálculos das taxas de letalidade. Todas as análises foram realizadas para o contexto do Brasil, das macrorregiões geográficas e das unidades da federação. Resultados: em relação a tendência de crescimento de novos casos o Brasil apresenta crescimento diário $23,8 \%$, a região norte é a que apresenta o maior incremento (29,6\%) e entre os estados, Amazonas e Maranhão são os que apresentam os maiores valores, respectivamente, $26,9 \%$ e $29,4 \%$. Observa-se que a taxa de letalidade no país é de 6,1\%, maior na região sudeste (6,7\%) e nos estados da Paraíba (13,9\%) e do Piauí (10,7\%). Conclusão: o Brasil apresenta crescimento diário significativo de novos casos e de óbitos em decorrência a COVID-19.

Palavras-chave: COVID-19, Pandemias, Brasil, Estudos de Séries Temporais

\section{ABSTRACT}

Objective: to analyze the temporal trend of cases and deaths due to COVID-19 that occurred in Brazil in the first 50 days of the epidemic in the country. Methods: this is an ecological time series study based on the records of new cases and deaths that occurred from February 26th to March 15th. To analyze the trend of new cases, Joinpoint Regression was performed, based on the accumulated daily rate, and calculations of lethality rates were also performed. All analyzes were performed for the context of Brazil, geographic macro-regions and federation units. Results: in relation to the growth trend of new cases, Brazil has a daily growth of $23.8 \%$, the north region is the one with the highest increase (29.6\%) and among the states, Amazonas and Maranhão are the ones that present the higher values, respectively, $26.9 \%$ and $29.4 \%$. It is observed that the lethality rate in the country is $6.1 \%$, higher in the southeast region (6.7\%) and in the states of Paraíba (13.9\%) and Piauí (10.7\%). Conclusion: Brazil has a significant daily growth of new cases and deaths due to COVID-19.

Keywords: COVID-19, Pandemics, Brazil, Time Series Studies 


\section{INTRODUÇÃO}

Desde o início do século XXI as autoridades globais de saúde pública têm centrado esforços no estudo sobre dois novos coronavírus que se tornaram agentes causadores das primeiras doenças do novo século e que representaram ameaça à saúde internacional devido ao seu potencial risco epidêmico. $\mathrm{O}$ coronavírus da Síndrome Respiratória Aguda Grave (Severe Acute Respiratory Syndrome Coronavirus - SARS-CoV) foi descoberto na China em 2001 e foi responsável por gerar uma epidemia global de Síndrome Respiratória Aguda Grave. Já o coronavírus da Síndrome Respiratória do Oriente Médio (Middle East Respiratory Syndrome - MERS-CoV), descoberto em 2012 na Arábia Saudita, foi responsável por uma alta letalidade decorrente da infecção grave no trato respiratório inferior ${ }^{1,2}$.

Em dezembro de 2019, a China notificou a ocorrência de 27 casos de pneumonia de etiologia desconhecida e 1 óbito, e em janeiro de 2020, após o isolamento do agente etiológico, verificou-se que se tratava de um novo coronavírus. O grupo de estudos em coronavírus do comitê internacional o denominou de coronavírus da Síndrome Respiratória Aguda Grave 2 (SARS-CoV-2), e a doença causada por este novo agente zoonótico foi denominada de Doença do Coronavírus - 2019 (Coronavirus Disease - COVID-2019) $)^{3,4}$.
Desde a sua descoberta, o novo coronavírus apresentou uma taxa de transmissão extremamente alta e em 30 de janeiro já haviam sido notificados 7.818 casos de pessoas infectadas em 18 países, e 170 pessoas já haviam falecido na China por conta da COVID-19, e frente a esta situação a Organização Mundial da Saúde (OMS) declarou situação de Emergência em Saúde Pública de Interesse Internacio$\mathrm{nal}^{5}$. Os casos de COVID-19 foram crescendo rapidamente em todo o mundo e em 11 de março a OMS decretou situação de pandemia, momento em que se registrava mais de 118 mil casos da doença em 113 países com mais de 4 mil óbitos ${ }^{6}$.

O primeiro registro da doença no Brasil ocorreu em 26 de fevereiro na cidade de São Paulo, e foi observada em um idoso com histórico de viagem a Itália ${ }^{7}$. Desde então os registros de novos casos da doença e de morte no país tem crescido significativamente, e, de acordo com os dados do Ministério da Saúde, até o dia 19 de abril há registro de mais de 36 mil casos e 2 mil mortes em todas as unidades da federação ${ }^{8}$. Frente a esta realidade fazse necessário uma análise epidemiológica dessa doença no Brasil com vistas à compreensão da tendência e da magnitude que a COVID-19 assumiu no Brasil.

Dessa forma, o objetivo deste estudo foi analisar a tendência temporal dos casos e óbitos por COVID-19 ocorridos no Brasil nos primeiros 50 dias da epidemia no país, analisando a expansão da doença por unidades da federação. 


\section{MÉTODOS}

Trata-se de um estudo ecológico, de serie temporal, no qual foram analisados os registros de novos casos de COVID-19 e de óbitos por esta doença, ocorridos de 26 de fevereiro a 15 de abril de 2020, com base nas informações disponibilizadas pelo Painel Coronavírus do Ministério da Saúde.

Para o cálculo da incidência acumulada diária foi considerada como denominador a população brasileira para o dia 15 de abril de 2020, segundo as informações de projeção da população para o Brasil e as unidades da federação disponibilizadas pelo Instituto Brasileiro de Geografia e Estatística (IBGE). Procedeuse também o cálculo taxa de mortalidade no período de 50 dias.

Para o cálculo da taxa de letalidade acumulada por COVID-19 no Brasil, adotou-se como parâmetro temporal a semana epidemiológica nacional. $\mathrm{O}$ cálculo da taxa de letalidade foi realizado pelo quociente entre o número total de óbitos e de casos registrados no último dia da semana de referência.

As análises foram realizadas para o contexto do Brasil, das macrorregiões geográficas e das unidades da federação. Para o cálculo da tendência temporal da incidência de COVID-19 realizou-se análise de regressão Joinpoint, em que o modelo final selecionado foi o modelo mais ajustado, com a Porcentagem Diária de Variação (Daily Percentage Change DPC) baseado na tendência de cada segmento, estimando se esses valores eram estatisticamente significativos $(\mathrm{p}<0,05)$. Para quantificar a tendência na coorte dos dias analisados, foi calculada a Média da Porcentagem Diária de Variação (Average Daily Percent Change - ADPC), com base na média geométrica acumulada das tendências do DPC, com pesos iguais para os comprimentos de cada segmento durante o intervalo fixado. Os testes de significância utilizados baseiam-se no método de permutação de Monte Carlo e no cálculo da variação percentual anual da razão, utilizando o logaritmo da razão ${ }^{9,10}$. As análises estatísticas foram realizadas utilizando-se o software Joinpoint Regression Program, versão 4.8.0.0.

\section{RESULTADOS}

Observa-se que a média de crescimento diário dos casos de COVID-19 no Brasil foi de 23,8\%, e, segundo a região geográfica, verifica-se que a região norte é a que apresentou o maior percentual de crescimento diário $(29,6 \%)$ seguida das regiões Sul (24,7\%) e Nordeste (24,6\%). A região sudeste, atual epicentro da doença no país, registrou um crescimento diário médio de $22,0 \%$ e a região Centro-Oeste é a que apresentou o menor crescimento $(20,5 \%)$ (tabela 1$)$.

O estado de São Paulo foi o primeiro a registrar a doença no país, em 26 de fevereiro, e Roraima foi o último estado, com a primeira notificação realizada em 22 de março.

Todos os estados apresentaram crescimento médio diário significativo dos casos de COVID-19 desde o primeiro registro, contudo, os estados do Rio Grande do Sul, Santa Catarina, Paraíba, São Paulo, Mato Grosso, Pernambuco, Rio de Janeiro, Pará, Ceará, Amapá, Amazonas e Maranhão apresentaram crescimento diário superior a $20 \%$, com destaque ao Maranhão que apresentou média de crescimento diário de 29,4\% (tabela 1). 
Tabela 1 - Tendência de crescimento dos casos de COVID-19 no Brasil nos primeiros 50 dias, de acordo com a região geográfica e Estado. Brasil, 2020.

\begin{tabular}{|c|c|c|c|c|c|c|c|}
\hline Região/Estado & $\begin{array}{c}\text { Data de regis- } \\
\text { tro do primeiro } \\
\text { caso }\end{array}$ & $\begin{array}{l}\text { Data de } \\
\text { registro do } \\
\text { último caso }\end{array}$ & $\begin{array}{l}\text { Número } \\
\text { de dias }\end{array}$ & $\begin{array}{l}\text { Número } \\
\text { de casos }\end{array}$ & ADPC & $\begin{array}{l}\text { Intervalo de Con- } \\
\text { fiança } 95 \%\end{array}$ & $p$ \\
\hline Brasil & $26 / 02 / 2020$ & $15 / 04 / 2020$ & 50 & 25.262 & $23,8^{\star}$ & $21,2-26,4$ & $<0,05$ \\
\hline Norte & $15 / 03 / 2020$ & $15 / 04 / 2020$ & 32 & 2.582 & $29,6^{*}$ & $23,8-35,6$ & $<0,05$ \\
\hline Rondônia & $20 / 03 / 2020$ & $15 / 04 / 2020$ & 27 & 69 & $18,1^{\star}$ & $14,5-21,8$ & $<0,05$ \\
\hline Acre & $19 / 03 / 2020$ & $15 / 04 / 2020$ & 28 & 101 & $13,6^{*}$ & $10,8-16,5$ & $<0,05$ \\
\hline Amazonas & $15 / 03 / 2020$ & $15 / 04 / 2020$ & 32 & 1.554 & $26,9^{*}$ & $22,9-31,1$ & $<0,05$ \\
\hline Roraima & $22 / 03 / 2020$ & $15 / 04 / 2020$ & 25 & 114 & $19,9^{*}$ & $16,6-23,2$ & $<0,05$ \\
\hline Pará & $19 / 03 / 2020$ & $15 / 04 / 2020$ & 28 & 384 & $23,5^{\star}$ & $21,0-25,9$ & $<0,05$ \\
\hline Amapá & $20 / 03 / 2020$ & $15 / 04 / 2020$ & 27 & 334 & $25,3^{*}$ & $18,6-32,3$ & $<0,05$ \\
\hline Tocantins & $19 / 03 / 2020$ & $15 / 04 / 2020$ & 28 & 26 & $14,5^{\star}$ & $12,7-16,4$ & $<0,05$ \\
\hline Nordeste & $06 / 03 / 2020$ & $15 / 04 / 2020$ & 41 & 6.184 & $24,6^{*}$ & $20,8-28,5$ & $<0,05$ \\
\hline Maranhão & $21 / 03 / 2020$ & $15 / 04 / 2020$ & 26 & 630 & $29,4^{\star}$ & $25,6-33,3$ & $<0,05$ \\
\hline Piauí & $20 / 03 / 2020$ & $15 / 04 / 2020$ & 27 & 75 & $12,4^{\star}$ & $10,9-13,9$ & $<0,05$ \\
\hline Ceará & $17 / 03 / 2020$ & $15 / 04 / 2020$ & 30 & 2.157 & $23,6^{*}$ & $21,4-25,9$ & $<0,05$ \\
\hline Rio Grande do Norte & $13 / 03 / 2020$ & $15 / 04 / 2020$ & 34 & 399 & $19,5^{*}$ & $15,0-24,1$ & $<0,05$ \\
\hline Paraíba & $19 / 03 / 2020$ & $15 / 04 / 2020$ & 28 & 151 & $20,2^{*}$ & $17,0-23,5$ & $<0,05$ \\
\hline Pernambuco & $12 / 03 / 2020$ & $15 / 04 / 2020$ & 35 & 1.484 & $21,8^{\star}$ & $19,2-24,5$ & $<0,05$ \\
\hline Alagoas & $08 / 03 / 2020$ & $15 / 04 / 2020$ & 39 & 82 & $11,8^{\star}$ & $9,7-13,8$ & $<0,05$ \\
\hline Sergipe & $15 / 03 / 2020$ & $15 / 04 / 2020$ & 32 & 46 & $14,3^{*}$ & $11,0-17,6$ & $<0,05$ \\
\hline Bahia & $06 / 03 / 2020$ & $15 / 04 / 2020$ & 41 & 807 & $16,9^{*}$ & $13,9-20,0$ & $<0,05$ \\
\hline Sudeste & $26 / 02 / 2020$ & $15 / 04 / 2020$ & 50 & 16.246 & $22,0^{*}$ & $19,5-24,5$ & $<0,05$ \\
\hline Minas Gerais & $08 / 03 / 2020$ & $15 / 04 / 2020$ & 39 & 903 & $19,7^{\star}$ & $16,4-23,2$ & $<0,05$ \\
\hline Espirito Santo & $06 / 03 / 2020$ & $15 / 04 / 2020$ & 41 & 557 & $17,2^{\star}$ & $14,9-19,5$ & $<0,05$ \\
\hline Rio de Janeiro & $05 / 03 / 2020$ & $15 / 04 / 2020$ & 42 & 3.743 & $23,1^{\star}$ & $21,0-25,3$ & $<0,05$ \\
\hline São Paulo & $26 / 02 / 2020$ & $15 / 04 / 2020$ & 50 & 11.043 & $21,1^{\star}$ & $17,9-24,3$ & $<0,05$ \\
\hline Sul & $10 / 03 / 2020$ & $15 / 04 / 2020$ & 37 & 2.403 & 24,7 & $23,0-26,5$ & $<0,05$ \\
\hline Paraná & $18 / 03 / 2020$ & $15 / 04 / 2020$ & 29 & 803 & $16,0^{*}$ & $14,2-17,8$ & $<0,05$ \\
\hline Santa Catarina & $13 / 03 / 2020$ & $15 / 04 / 2020$ & 34 & 853 & $20,1^{\star}$ & $18,8-21,4$ & $<0,05$ \\
\hline Rio Grande do Sul & $10 / 03 / 2020$ & $15 / 04 / 2020$ & 37 & 747 & $20,1^{\star}$ & $17,8-22,4$ & $<0,05$ \\
\hline Centro-Oeste & $07 / 03 / 2020$ & $15 / 04 / 2020$ & 40 & 1.258 & $20,3^{\star}$ & $18,6-22,0$ & $<0,05$ \\
\hline Mato Grosso do Sul & $16 / 03 / 2020$ & $15 / 04 / 2020$ & 31 & 121 & $14,6^{*}$ & $12,7-16,5$ & $<0,05$ \\
\hline Mato Grosso & $20 / 03 / 2020$ & $15 / 04 / 2020$ & 27 & 151 & $21,5^{\star}$ & $18,0-25,1$ & $<0,05$ \\
\hline Goiás & $13 / 03 / 2020$ & $15 / 04 / 2020$ & 34 & 304 & $15,1^{\star}$ & $13,1-17,2$ & $<0,05$ \\
\hline Distrito Federal & $07 / 03 / 2020$ & $15 / 04 / 2020$ & 40 & 682 & $18,8^{*}$ & $16,7-21,0$ & $<0,05$ \\
\hline
\end{tabular}


Figura 1 - Curva da Taxa de Letalidade acumulada da COVID-19 no Brasil de acordo com a semana epidemio- Medeiros et al lógica nacional, a partir do primeiro óbito registrado.

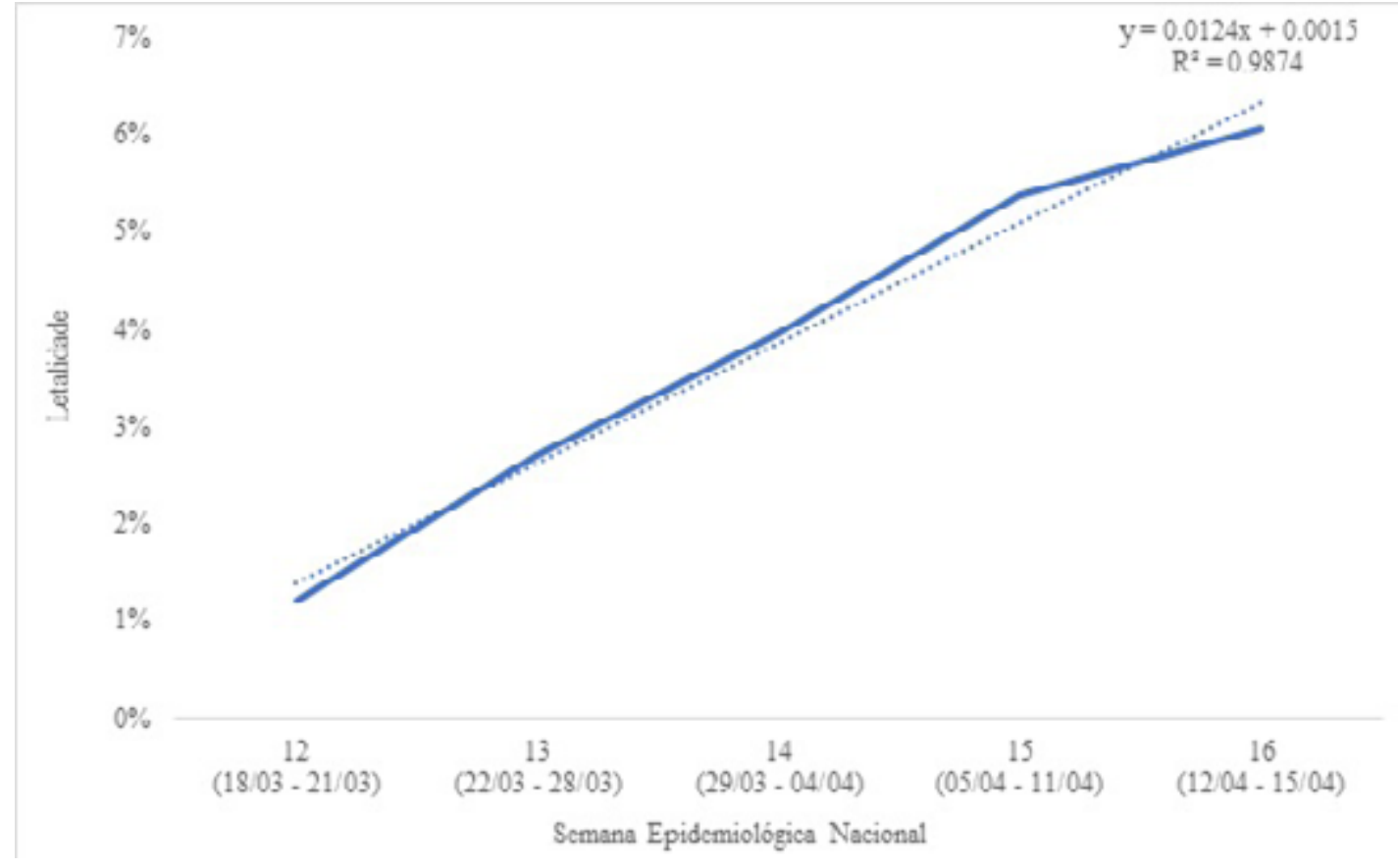

Na figura 1 é possível observar a curva da letalidade acumulada da COVID-19 no Brasil, de acordo com a semana epidemiológica. A reta da regressão apresenta o $\beta 0$ de 0,012 e $\mathrm{R}^{2}$ de 0,98 indicando a tendência temporal de aumento.

No período analisado a taxa de letalidade acumulada no Brasil foi 6,1\%. A maior taxa foi observada na região sudeste $(6,7 \%)$ e a menor na região Centro-Oeste $(1,7 \%)$. Ao analisar por estado observa-se que a maior taxa de letalidade entre as unidades da federação foi na $\mathrm{Pa}$ raíba (13,9\%), em 15 dias após o registro do primeiro óbito, seguida por Piauí com uma taxa de 10,7\% em 19 dias desde a primeira notificação (tabela 2).

\section{DISCUSSÃO}

Os resultados indicam que, apesar das unidades da federação terem adotado a utilização de medidas não farmacológicas comunitárias para o controle da doença a partir de 13 de março, o Brasil enfrenta um crescimento significativo dos casos de COVID-19 e de mortes em decorrência a esta nova doença.

Ao comparar o Brasil com a China, no mesmo período, verifica-se que a média de crescimento diário de novos casos no país é 8 vezes maior do que a registrada na China, e a taxa de letalidade é quase o dobro, segundo o estudo de Lima, Medeiros e Barbosa ${ }^{11}$. O estudo apontou que a China apresentou crescimento significativo de novos casos nos primeiros 16 dias e na sequência houve tendência significativa de redução com média de crescimento diário de $-3,4 \%$ e taxa de letalidade de 3,95\%.

A análise da taxa de letalidade de outros países, no mesmo período, aponta que o Brasil apresenta taxa superior a observada na Espanha (5,01\%), nos Estados Unidos (2,93\%), na França $(2,17 \%)$ e no Reino Unido (4,44\%), e menor que a da Itália $(8,30 \%)^{12-15}$. 
Tabela 2 - Descrição dos óbitos por COVID-19 no Brasil nos primeiros 50 dias, de acordo com a região geográfica e Estado. Brasil, 2020.

\begin{tabular}{|c|c|c|c|c|c|}
\hline Região/Estado & População & $\begin{array}{c}\text { Data de registro } \\
\text { do primeiro } \\
\text { óbito }\end{array}$ & $\begin{array}{c}\text { Número de } \\
\text { óbitos }\end{array}$ & Letalidade & Mortalidade \\
\hline Brasil & 211.394 .400 & $18 / 03 / 2020$ & 1.532 & $6,1 \%$ & 0,725 \\
\hline Norte & 18.613 .064 & $25 / 03 / 2020$ & 143 & $5,5 \%$ & 0,768 \\
\hline Rondônia & 1.792 .207 & $31 / 03 / 2020$ & 2 & $5,7 \%$ & 0,112 \\
\hline Acre & 892.012 & $07 / 04 / 2020$ & 3 & $3,0 \%$ & 0,336 \\
\hline Amazonas & 4.194 .489 & $25 / 03 / 2020$ & 106 & $6,8 \%$ & 2,527 \\
\hline Roraima & 615.882 & $04 / 04 / 2020$ & 3 & $2,6 \%$ & 0,487 \\
\hline Pará & 8.673 .422 & $01 / 04 / 2020$ & 21 & $5,5 \%$ & 0,242 \\
\hline Amapá & 858.280 & $04 / 04 / 2020$ & 7 & $2,1 \%$ & 0,816 \\
\hline Tocantins & 1.586 .772 & $15 / 04 / 2020$ & 1 & $3,8 \%$ & 0,063 \\
\hline Nordeste & 57.310 .427 & $25 / 03 / 2020$ & 392 & $6,3 \%$ & 0,684 \\
\hline Maranhão & 7.106 .572 & $30 / 03 / 2020$ & 34 & $5,4 \%$ & 0,478 \\
\hline Piauí & 3.278 .914 & $28 / 03 / 2020$ & 8 & $10,7 \%$ & 0,244 \\
\hline Ceará & 9.175 .609 & $26 / 03 / 2020$ & 116 & $5,4 \%$ & 1,264 \\
\hline Rio Grande do Norte & 3.528 .945 & $29 / 03 / 2020$ & 19 & $4,8 \%$ & 0,538 \\
\hline Paraíba & 4.033 .697 & $01 / 04 / 2020$ & 21 & $13,9 \%$ & 0,521 \\
\hline Pernambuco & 9.605 .269 & $25 / 03 / 2020$ & 143 & $9,6 \%$ & 1,489 \\
\hline Alagoas & 3.348 .768 & $31 / 03 / 2020$ & 5 & $6,1 \%$ & 0,149 \\
\hline Sergipe & 2.314 .847 & $02 / 04 / 2020$ & 4 & $8,7 \%$ & 0,173 \\
\hline Bahia & 14.917 .806 & $29 / 03 / 2020$ & 27 & $3,3 \%$ & 0,181 \\
\hline Sudeste & 88.870 .014 & $17 / 03 / 2020$ & 1.091 & $6,7 \%$ & 1,228 \\
\hline Minas Gerais & 21.266 .053 & $30 / 03 / 2020$ & 30 & $3,3 \%$ & 0,141 \\
\hline Espirito Santo & 4.054 .096 & $02 / 04 / 2020$ & 18 & $3,2 \%$ & 0,444 \\
\hline Rio de Janeiro & 17.341 .695 & $19 / 03 / 2020$ & 265 & $7,1 \%$ & 1,528 \\
\hline São Paulo & 46.208 .168 & $17 / 03 / 2020$ & 778 & $7,0 \%$ & 1,684 \\
\hline Sul & 30.142 .696 & $25 / 03 / 2020$ & 85 & $3,5 \%$ & 0,282 \\
\hline Paraná & 11.497 .918 & $27 / 03 / 2020$ & 38 & $4,7 \%$ & 0,330 \\
\hline Santa Catarina & 7.232 .669 & $26 / 03 / 2020$ & 28 & $3,3 \%$ & 0,387 \\
\hline Rio Grande do Sul & 11.412 .109 & $25 / 03 / 2020$ & 19 & $2,5 \%$ & 0,166 \\
\hline Centro-Oeste & 16.458 .199 & $26 / 03 / 2020$ & 40 & $1,7 \%$ & 0,073 \\
\hline Mato Grosso do Sul & 2.802 .617 & $31 / 03 / 2020$ & 4 & $3,3 \%$ & 0,143 \\
\hline Mato Grosso & 3.516 .474 & $03 / 04 / 2020$ & 4 & $2,6 \%$ & 0,114 \\
\hline Goiás & 7.095 .188 & $26 / 03 / 2020$ & 15 & $4,9 \%$ & 0,211 \\
\hline Distrito Federal & 3.043 .920 & $29 / 03 / 2020$ & 17 & $2,5 \%$ & 0,558 \\
\hline
\end{tabular}

"Taxa de mortalidade por 100 mil habitantes. 
Acredita-se que a atual situação do Brasil possa ser explicada pela baixa adesão da população as orientações de distanciamento social, visto que foi possível verificar maior adesão a esta medida nos primeiros dias após as determinações dos governos estaduais e municipais, e observou-se um maior fluxo de pessoas nas ruas com o passar dos dias. O Centro de Controle e Prevenção de Doenças (CDC) dos Estados Unidos sugere que para a efetividade da adoção de intervenções não farmacológicas durante uma pandemia deve haver aceitação e participação da população ${ }^{16}$. Os pronunciamentos do presidente da república, contraditório aos posicionamentos do ministério da saúde e dos governos estaduais e municipais, podem ter contribuído para esta baixa adesão. No atual curso da epidemia de COVID-10 no Brasil é fundamental que a comunicação das autoridades com a população seja honesta e transparente, para que haja confiança da população, e consequentemente, adotem as medidas de controle para mitigação da doença ${ }^{17,18}$.

O desconhecimento acerca desta nova doença impõe uma série de questionamentos quanto a adoção destas medidas no que se refere, principalmente, ao momento adequando de implementação e de suspensão das mesmas. O CDC dos Estados Unidos, com base nos documentos sobre a influenza pandêmica, indica que o momento adequado para a implementação das intervenções não farmacológicas comunitárias deve ser baseado na severidade da doença ${ }^{17}$.

Conforme abordado por Barreto e colaboradores ${ }^{19}$, apesar de reconhecer a necessidade e a importância da adoção de medidas de distanciamento social, estas não podem ter uma vigência muito longa, diante das questões sociais, econômicas e educacionais impostas, contudo, o relaxamento precoce pode caracterizar-se como um desafio tão, ou mais, complicado que a sua própria implementação.

Ainda, por se tratar de uma condição respiratória aguda grave, as estratégias utilizadas em situações semelhantes ${ }^{20,21}$ podem ser tomadas como balizadores para o momento atual, assim como as intervenções realizadas na China para o controle desta doença ${ }^{22,23}$.

Os resultados de uma recente revisão de literatura ${ }^{24}$ sugerem que o controle da epidemia deva ser pautado na implementação de estratégias articuladas intersetorialmente e coordenadas entre as diferentes esferas governamentais, com base no isolamento de casos, quarentena de contatos, medidas amplas de distanciamento social - com expectativa de redução dos contatos em no mínimo 60\%, e fortalecimento das ações do sistema de vigilância nos três níveis de atenção à saúde, incluindo a ampliação da capacidade de testagem.

Frente a esta situação, se faz necessário salientar que as informações públicas disponíveis quanto ao número de casos referem-se aos casos mais graves da doença, conforme descrito no boletim epidemiológico do Ministério da Saúde do dia 17 de abril ${ }^{25}$, o qual orienta que os testes laboratoriais devam ser direcionados aos profissionais da saúde e da segurança e para o diagnóstico dos casos graves e de óbitos, demonstrando claramente um cenário de subnotificação dos casos o que torna o desconhecimento sobre a real extensão da doença no país um desafio para o estabelecimento de estratégias a curto e médio prazo. 
Associado ao cenário de desconhecimento da doença no território nacional é imprescindível lembrar que há evidências de que pessoas assintomáticas podem ser capazes de transmitir o vírus, o que torna o controle ainda mais difícil, sendo, portanto, mais uma justificativa para a ampla realização de testes, com sensibilidade e especificidade adequados ${ }^{26}$

A ampliação da testagem para COVID-19 se constitui como importante ferramenta para o reconhecimento real da epidemia no Brasil e para a implementação de intervenções específicas ${ }^{27}$.

Tal sustentação referente a necessidade de ampliação na realização de testes sustenta-se com o estudo realizado por Bastos e colaboradores ${ }^{28}$, que teve como objetivo investigar o padrão das hospitalizações ocorridas no país por Síndromes Respiratórias Agudas Graves (SRAG) após a primeira notificação por contaminação do SARS-CoV-2 em comparação aos anos de 2010 a 2019, e identificou que as internações por esta condição em 2020 foi superior ao observado em todo o período analisado, sugerindo, portanto, que a COVID-19 está sendo identificada pelo sistema de vigilância de SRAG, tendo em vista a elevação no número de internações por SRAG associado ao desconhecimento do agente etiológico das hospitalizações e a maior prevalência entre idosos, embora não haja comprovação diante da ausência de testes.

O cenário atual, no qual observase o crescimento diário dos casos de COVID-19 associado a elevação das internações por SRAG, evidencia a necessidade de empenho de todos os setores na tentativa de minimizar a sobrecarga do sistema de saúde, tendo em vista que alguns estados já apresentam mais de $90 \%$ de ocupação dos leitos de UTI em instituições públicas, como é o caso do Amazonas, Pará, Pernambuco e Rio de Janeiro ${ }^{29}$, indicando que estes estados se aproximam da situação de colapso no sistema de saúde.

Sabe-se que historicamente as hospitalizações por SRAG acontecem, majoritariamente, no outono e inverno ${ }^{28}$, assim, torna-se clara a necessidade da ampliação do monitoramento da população, em especial da população idosa, quanto as SRAG e as síndromes gripas (SG) com vistas a reduzir as internações em decorrência estas condições, e consequentemente, contribuir para evitar o colapso do sistema de saúde.

Nesta perspectiva, a atuação dos profissionais da atenção primária à saúde é primordial para que se obtenha êxito no monitoramento da população. Contudo, é necessário que o processo de trabalho dos profissionais de saúde seja revisitado, em especial dos agentes comunitários de saúde. Neste momento de pandemia, em que o distanciamento social se configura como estratégia prioritária para o controle da doença, a incorporação de novas estratégias de monitoramento em saúde, incluindo as tecnologias de informação e comunicação, apresenta-se como importante ferramenta para a efetivação do cuidado diante dos potenciais benefícios como melhora da comunicação e coordenação do cuidado ${ }^{30}$. 


\section{CONCLUSÃO}

A análise dos primeiros 50 dias da epidemia de COVID-19 no Brasil evidencia que todos os estados apresentaram crescimento diário significativo de novos casos, sendo que os estados do Amazonas e Maranhão foram os que registraram o maior crescimento. A taxa de letalidade do Brasil é superior à verificada em outros países no mesmo período, e a Paraíba e o Piauí foram os estados com a maior letalidade. Os resultados apontam a necessidade de ampliação da realização dos testes diagnósticos para o reconhecimento real da epidemia no país, e reforçam a necessidade de manutenção das intervenções não farmacológicas comunitárias para a mitigação da doença.

\section{REFERÊNCIAS}

1. Hui DS, Azhar EI, Kim YJ, Memish ZA, Oh MD, Zumia A. Middle East respiratory syndrome coronavirus: risk factors and determinants of primary, household, and nosocomial transmission. Lancet Infect Dis 2018; 18: e217-27. doi: 10.1016/S14733099(18)30127-0

2. Azhar E.I., Lanini S., Ippolito G., Zumla A. (2016) The Middle East Respiratory Syndrome Coronavirus - A Continuing Risk to Global Health Security. In: Rezza G., Ippolito G. (eds) Emerging and Re-emerging Viral Infections. Advances in Experimental Medicine and Biology, vol 972. Springer, Cham

3. Guo YR, Cao QD, Hong ZS, Tan YY, Chen SD, Jin HJ, et al. The origin, transmission and clinical therapies on coronavirus disease 2019 (COVID-19) outbreak - an update on the status. Military Medical Research. 2020; 7:11. doi: 10.1186/ s40779-020-00240-0
4. Chan JFW, Yuan S, Kok KH, To KKW, Chu H, Yang J, et al. A familial cluster of pneumonia associated with the 2019 novel coronavirus indicating person-to-person transmission: a study of a family cluster. Lancet. 2020; 395: 514-23. doi: 10.1016/ S0140-6736(20)30154-9

5. World Health Organization. Coronavirus disease 2019 (COVID-19). Situation Report - 10 [Internet]. 2020 [Acesso em 2020 mar 18]. Disponível em: https://www.who.int/ emergencies/diseases/novel-coronavirus-2019/situation-reports/

6. World Health Organization. Coronavirus disease 2019 (COVID-19). Situation Report - 51 [Internet]. 2020 [Acesso em 2020 mar 18]. Disponível em: https://www.who.int/ emergencies/diseases/novel-coronavirus-2019/situation-reports/

7. Croda J, Oliveira WK, Frutuoso RL, Mandetta LH, Baia-da-Silva DC, Brito-Sousa JD, et al. COVID-19 in Brazil: advantages of a socialized unified health system and preparation to contain cases. Journal of the Brazilian Society of Tropical Medicine. 2020; 53:e20200167. doi: 10.1590/00378682-0167-2020

8. Brasil. Ministério da Saúde. Secretaria de Vigilância em Saúde. Boletim Epidemiológico 12 [Internet]. 2020 [Acesso em 2020 abr 19]. Disponível em: https://portalarquivos.saude.gov. br/images/pdf/2020/April/19/BE12Boletim-do-COE.pdf

9. Kim HJ, Fay MP, Feuer EJ, Midthune DN. Permutation tests for joinpoint regression with applications to cancer rates. Stat Med. 2000;19:335351. doi: 10.1002/(sici)1097$0258(20000215) 19: 3<335:$ :aid $\operatorname{sim} 336>3.0 . \operatorname{co} ; 2-\mathrm{z}$

10. Kim HJ, Fay MP, Yu B, Barrett MB, Feuer EJ. Comparability of Segmented Line Regression Models. Biometrics. 2004; 60(4):1005-1014. doi: 10.1111/j.0006-341X.2004.00256.X 
11. Lima KC, Medeiros AA, Barbosa IR. Análise da tendência de casos e óbitos por Coronavirus Disease-2019 (COVID-19) nos principais países afetados e no Brasil: uma análise dos primeiros 50 dias da pandemia. J. Health Biol Sci. 2020;8(1):1-5. doi: 10.12662/2317-3219jhbs.v8i1.3233. p1-6.2020

12. World Health Organization. Coronavirus disease 2019 (COVID-19). Situation Report - 52 [Internet]. 2020 [Acesso em 2020 abr 25]. Disponível em: https://www.who.int/ emergencies/diseases/novel-coronavirus-2019/situation-reports/

13. World Health Organization. Coronavirus disease 2019 (COVID-19). Situation Report - 54 [Internet]. 2020 [Acesso em 2020 abr 25]. Disponível em: https://www.who.int/ emergencies/diseases/novel-coronavirus-2019/situation-reports/

14. World Health Organization. Coronavirus disease 2019 (COVID-19). Situation Report - 60 [Internet]. 2020 [Acesso em 2020 abr 25]. Disponível em: https://www.who.int/ emergencies/diseases/novel-coronavirus-2019/situation-reports/

15. World Health Organization. Coronavirus disease 2019 (COVID-19). Situation Report - 61 [Internet]. 2020 [Acesso em 2020 abr 25]. Disponível em: https://www.who.int/ emergencies/diseases/novel-coronavirus-2019/situation-reports/

16. Qualls N, Levitt A, Kanade N, et al. Diretrizes da comunidade para mitigação de prevenção da gripe pandêmica - Estados Unidos, 2017. MMWR Recommend Rep 2017; 66 (No RR-1): 1-34. doi: 10.15585/ mmwr.rr6601al
17. Garcia LP, Duarte E. Intervenções não farmacológicas para o enfrentamento à epidemia da COVID-19 no Brasil. Epidemiol Serv Saude 2020; 29(2):e2020222. doi: $10.5123 /$ S167949742020000200009

18. Bezerra A, Silva CEM, Soares FRG, Silva JAM. Fatores associados ao comportamento da população durante $\mathrm{o}$ isolamento social na pandemia de COVID-19. Ciênc saúde coletiva 2020. [Acesso em 2020 Abr 26]. Disponível em: http:// www.cienciaesaudecoletiva.com. br/novidades/fatores-associados-ao-comportamento-da-populacao-durante-o-isolamento-social-na-pandemia-de-covid19/105

19. Barreto ML, Barros AJD, Carvalho MS, Codeço CT, Hallal PRC, Medronho RA, et al. O que é urgente e necessário para subsidiar as políticas de enfrentamento da pandemia de COVID-19 no Brasil? Rev Bras Epidemiol 2020; 23: E200032. doi: 10.1590/1980-549720200032

20. Markel H, Stern AM, Navarro JA, Michalsen JR, Monto AS, Di Giovanni C. Nonpharmaceutical influenza mitigation strategies, US communities, 1918-1920 pandemic. Emerg Infect Dis 2006; 12(12):1961-4. doi: 10.3201/eid1212.060506

21. Teh B, Olsen K, Black J, Cheng AC, Aboltins C, Bull K, et al. Impact of swine influenza and quarantine measures on patients and households during the H1N1/09 pandemic. Scand J Infect Dis 2012; 44(4):289-96. doi: 10.3109/00365548.2011.631572. 
22. Chen $\mathrm{X}, \mathrm{Yu}$ B. First two months of the 2019 Coronavirus Disease (COVID-19) epidemic in China: realtime surveillance and evaluation with a second derivative model. Global Health Research and Policy 2020, 5:7; 1-9. https://doi.org/10.1186/s41256020-00137-4

23. Kraemer MU, Yang CH, Gutierrez B, Wu CH, Klein B, Pigott DM, et al. The effect of human mobility and control measures on the COVID-19 epidemic in China. Science 2020: eabb4218.

24. Aquino E, Silveira IH, Pescarini J, Aquino R, Souza-Filho JA. Medidas de distanciamento social no controle da pandemia de COVID-19: Potenciais impactos e desafios no Brasil. [Acesso em 2020 Abr 27]. Disponível em: http:// www.cienciaesaudecoletiva.com.br/ novidades/medidas-de-distanciamento-social-no-controle-da-pandemia-de-covid19-potenciais-impactos-e-desafios-no-brasil/103

25. Brasil. Ministério da Saúde. Secretaria de Vigilância em Saúde. Boletim Epidemiológico 11 [Internet]. 2020 [Acesso em 2020 abr 24]. Disponível em: https://www.saude.gov.br/images/pdf/2020/April/18/2020-04-17--BE11---Boletim-do-COE-21h.pdf

26. Rothe C, Schunk M, Sothmann P, Bretzel G, Froeschl G, Wallrauch C, et al. Transmission of 2019-nCoV Infection from an asymptomatic contact in Germany. N Engl J Med. 2020; 382(10): 970-1. doi: 10.1056/ NEJMc2001468.
27. Villela DAM. The value of mitigating epidemic peaks of COVID-19 for more effective public health responses. Rev Soc Bras Med Trop 2020; 53:e20200135. doi: 10.1590/00378682-0135-2020

28. Bastos LS, Niquini RP, Lana RM, Villela DAM, Cruz OG, Coleho FC, et al. COVID-19 e hospitalizações por SRAG no Brasil: uma comparação até a 12 a semana epidemiológica de 2020. Cad. Saúde Pública 2020; 36(4):e0007012. doi: 10.1590/0102$311 \mathrm{X} 00070120$

29. Oliveira C. 'A gente está no limite e vai piorar', alerta sanitarista da UFRJ sobre covid-19 no Brasil. Rede Brasil Atual; [Internet] 2020 Abr 25. [Acesso em 2020 abr 27]. Disponível em: https://www.redebrasilatual.com.br/ saude-e-ciencia/2020/04/a-gente-esta-no-limite-e-vai-piorar-alerta-sanitarista-da-ufrj-sobre-covid-19-nobrasil/

30. Santos AF, Sobrinho DF, Araujo LL, Procópio CSD, Lopes EAS, Lima AMLD, et al. Incorporação de Tecnologias de Informação e Comunicação e qualidade na atenção básica em saúde no Brasil. Cad Saúde Pública 2017; 33(5): e00172815 doi: 10.1590/0102-311X00172815

Recebido: 29/04/2020

Aprovado: 25/05/2020 